\title{
Three parameter Transmuted Rayleigh distribution with application to Reliability data
}

\author{
Muhammad Shuaib Khan ${ }^{*}$ \\ School of Mathematical and Physical Sciences, University of Newcastle \\ Callaghan, NSW 2308, Australia \\ Muhammad.S.Khan@newcastle.edu.au \\ Robert King \\ School of Mathematical and Physical Sciences, University of Newcastle \\ Callaghan, NSW 2308, Australia \\ robert.king@newcastle.edu.au \\ Irene Lena Hudson \\ School of Mathematical and Physical Sciences, University of Newcastle \\ Callaghan, NSW 2308, Australia \\ Irene.Hudson@newcastle.edu.au
}

Received 12 September 2015

Accepted 3 March 2016

\begin{abstract}
This research introduces the three parameter transmuted Rayleigh distribution with an application to fatigue fracture data. Using the quadratic rank transmutation map method proposed by Shaw et al. [25] we develop the three parameter transmuted Rayleigh distribution. This research also introduces the new class of weighted Rayleigh distribution by using Azzalini [2] method. Some structural properties of the new distribution are derived such as moments, incomplete moments, probability weighted moments, moment generating function, entropies, mean deviation and the $k^{\text {th }}$ moment of order statistics. The parameters of the proposed model are estimated using the maximum likelihood estimation and obtain the observed information matrix. The potentiality of the proposed model is illustrated using fatigue fracture data.
\end{abstract}

Keywords: 2P-Rayleigh distribution; weighted Rayleigh distribution; moment estimation; order statistics; maximum likelihood estimation.

2000 Mathematics Subject Classification: 90B25, 62N05

\section{Introduction}

The Rayleigh distribution is a well-known lifetime distribution introduced by Rayleigh, L [23] and it has been extensively used for modelling data in different areas of engineering and medical sciences. The Rayleigh

\footnotetext{
* Corresponding author.
} 
distribution is the special case of Weibull distribution, is widely used to model events data. The one parameter Rayleigh distributional properties, estimation and characterisations have been studied by many authors, such as Johnson, Kotz and Balakrishnan [10], Dey and Das [6], Dey [5]. More recently Sanku, Tanujit and Kundu [24] and Khan, Provost and Singh [16] studied the two-parameter Rayleigh distribution, which has one scale and one location parameter has the following reliability function as

$$
R(x ; \alpha, \mu)=\exp \left(-\alpha(x-\mu)^{2}\right), \quad(x>\mu),
$$

The cumulative distribution function, probability density function and quantile function corresponding to (1) are given by

$$
\begin{aligned}
& G(x ; \alpha, \mu)=1-\exp \left(-\alpha(x-\mu)^{2}\right), \quad(x>\mu), \\
& g(x ; \alpha, \mu)=2 \alpha(x-\mu) \exp \left(-\alpha(x-\mu)^{2}\right)
\end{aligned}
$$

and

$$
Q(p)=\mu+\left\{-\frac{1}{\alpha} \ln (1-p)\right\}^{1 / 2}, \quad 0 \leq p \leq 1
$$

respectively. The median is given by

$$
\operatorname{Median}(X)=\mu+\left\{-\frac{1}{\alpha} \ln \left(\frac{1}{2}\right)\right\}^{1 / 2}
$$

The hazard function is given by

$$
h(x ; \alpha, \mu)=\frac{2 \alpha(x-\mu) \exp \left(-\alpha(x-\mu)^{2}\right)}{1-\exp \left(-\alpha(x-\mu)^{2}\right)},
$$

respectively. where $\alpha>0$ is the scale parameter and $\mu$ is the location parameter. The two parameter Rayleigh distribution approches to the one parameter classical Rayleigh model when location parameter $\mu=0$. Figure 1 displays densities with histogram for two simulated data sets, using equation (4) for the two parameter Rayleigh distribution

Gupta and Kundu [8] introduced the new class of weighted exponential distribution using the Azzalini [2] method. For the comparison purpose we introduce the weighted Rayleigh (WR) distribution. The two parameter weighted Rayleigh distribution with parameters $\alpha, \lambda>0$, for $x>0$, its (cdf) is given by

$$
F(x ; \alpha, \lambda)=\frac{\alpha+1}{\alpha}\left[1-\exp \left(-\lambda x^{2}\right)-\frac{1}{1+\alpha}\left\{1-\exp \left(-(\alpha+1) \lambda x^{2}\right)\right\}\right],
$$

where $\alpha>0$ and $\lambda>0$ are the scale parameters, respectively. The probability density function (pdf) corresponding to (6) is

$$
f(x ; \alpha, \lambda)=\frac{\alpha+1}{\alpha} 2 \lambda x \exp \left(-\lambda x^{2}\right)\left\{1-\exp \left(-\alpha \lambda x^{2}\right)\right\}
$$

As $\alpha \rightarrow \infty$, WR $(\alpha)$ converges to the standard Rayleigh distribution. So the Rayleigh distribution can be obtained as a special case of the WR distribution. The $k^{t h}$ moment $(k>0)$ of the WR distribution is given by

$$
\dot{\mu}_{k}=\frac{\alpha+1}{\alpha} \frac{\Gamma\left(\frac{k}{2}+1\right)}{\lambda^{\frac{k}{2}}}\left\{1-\frac{1}{(\alpha+1)^{\frac{k}{2}+1}}\right\}
$$


The $k^{\text {th }}$ moment of the weighted Rayleigh distribution is in closed form expression. The important features and characteristics of the weighted Rayleigh distribution can be studied through moments, furthermore the bathtub shape property, reliability behaviour, mean, variance, coefficient of variation, coefficient of skewness and coefficient of kurtosis can be analysed numerically using statistical software.

(a)

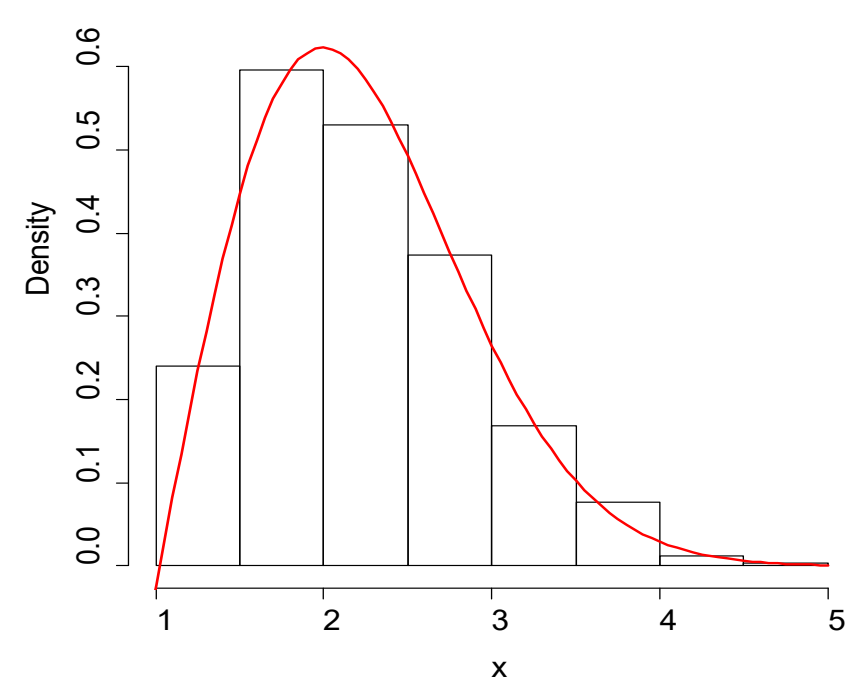

(b)

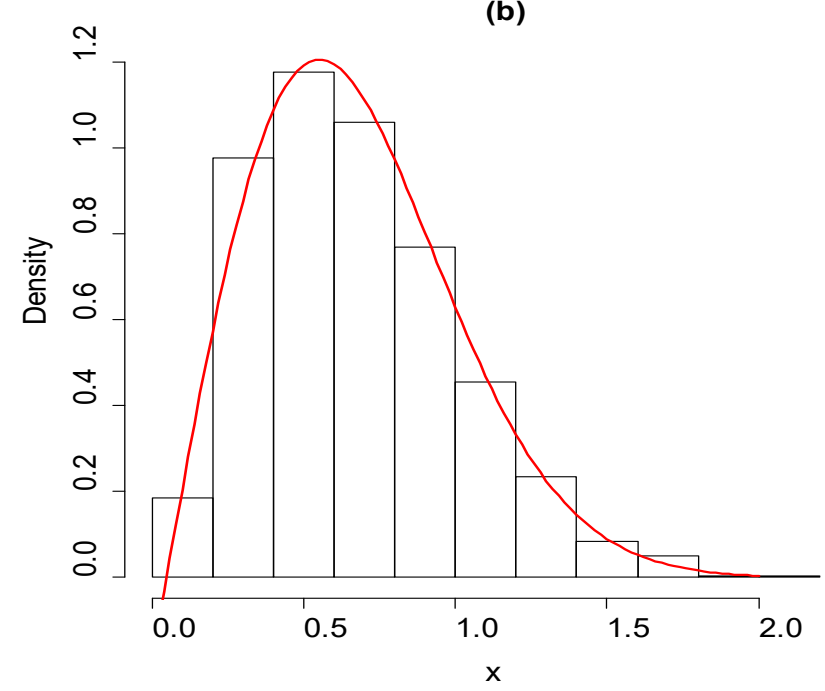

Fig. 1. Plots of the two parameter Rayleigh densities for simulated data sets,

(a) $\alpha=0.5 ; \mu=1$ (b) $\alpha=2 ; \mu=0.05$.

Recently sevaral distributions have been proposed from the transmuted family of lifetime distributions. Aryal et al. [3, 4] proposed the transmuted extreme value and transmuted Weibull distributions and examined various structural properties of these models with applications. Khan and King [11, 12] studied the transmuted modified Weibull distribution and the transmuted generalized Inverse Weibull distribution and discussed some theoretical properties of these distributions. Recently Khan and King [13, 14] proposed the 
transmuted Inverse Weibull and transmuted modified Inverse Rayleigh distributions for modelling reliability data. More recently Khan et al. [15] proposed the transmuted Chen distribution and investigated various structural properties with an application to reliability data. Recently Merovci [18] studied the transmuted Rayleigh distribution with application to balder cancer data. Merovci [19] studied the transmuted generalized Rayleigh distribution. Yuzhu et al. [27] proposed and studied the transmuted linear exponential distribution and discussed some of its structural properties. Elbatal. et al [7] introduced the transmuted generalized linear exponential distribution and formulated some structural properties of this distribution.

By using the quadratic rank transmutation map technique proposed by Shaw et al. [25], we develop the three-parameter transmuted Rayleigh distribution. According to this approach a random variable $X$ is said to have a transmuted distribution if its cumulative distribution function (cdf) satisfies the following relationship

$$
F(x)=(1+\lambda) G(x)-\lambda G(x)^{2}, \quad|\lambda| \leq 1
$$

and

$$
f(x)=g(x)\{(1+\lambda)-2 \lambda G(x)\},
$$

where $G(x)$ is the cdf of the base distribution, $g(x)$ and $f(x)$ are the corresponding probability density functions (pdf) associated with $G(x)$ and $F(x)$, respectively. It is important to note that at $\lambda=0$ we have the distribution of the base random variable. The paper is organised as follows, in Section 2, we present the analytical shapes of the probability density and hazard function of the three parameter transmuted Rayleigh distribution. We demonstrate the quantile functions, moment estimation, incomplete moment, probability weighted moment and moment generating functions in Section 3. Entropies and mean deviations are formulated in Section 4. The $k^{\text {th }}$ moments of $r^{\text {th }}$ order statistics are derived in Section 5. Maximum likelihood estimates (MLEs) of the unknown parameters are discussed in Section 6 and evaluate the performance of the MLEs using simulations in Section 7. The flexibility and utility of the proposed model is emphasized in Section 8 by using it to model the fatigue fracture data. Concluding remarks are addressed in Section 9.

\section{Transmuted Rayleigh distribution}

A random variable $X$ is said to have transmuted Rayleigh distribution with three parameters $\alpha>0,|\lambda| \leq 1$ and $X>\mu$. It can be used to represent the failure probability density function given by

$$
f(x ; \alpha, \mu, \lambda)=2 \alpha(x-\mu) \exp \left(-\alpha(x-\mu)^{2}\right)\left\{1-\lambda+2 \lambda \exp \left(-\alpha(x-\mu)^{2}\right)\right\},
$$

The cumulative distribution function corresponding to (11) is

$$
F(x ; \alpha, \mu, \lambda)=\left\{1-\exp \left(-\alpha(x-\mu)^{2}\right)\right\}\left\{1+\lambda \exp \left(-\alpha(x-\mu)^{2}\right)\right\} .
$$

The three parameter transmuted Rayleigh distribution approaches the two parameter transmuted Rayleigh distribution when the location parameter $\mu=0$. When the transmuting parameter $\lambda=0$ then the subject distribution approaches to the base model. Here $\alpha$ is the scale parameter, $\mu$ is the location parameter and $\lambda$ is the transmuted parameter which provides more flexibility in the propose distribution. Plots of the densities and hazard functions of the three parameter transmuted Rayleigh distribution are displayed in Figure 2 with 
different choices of the parameters. If $X \sim \operatorname{TR}(x ; \alpha, \mu, \lambda)$ then the reliability function (RF), hazard function and cumulative hazard function corresponding to (11) are given by

$$
\begin{gathered}
R(x ; \alpha, \mu, \lambda)=1-\left\{1-\exp \left(-\alpha(x-\mu)^{2}\right)\right\}\left\{1+\lambda \exp \left(-\alpha(x-\mu)^{2}\right)\right\}, \\
h(x ; \alpha, \mu, \lambda)=\frac{2 \alpha(x-\mu) \exp \left(-\alpha(x-\mu)^{2}\right)\left\{1-\lambda+2 \lambda \exp \left(-\alpha(x-\mu)^{2}\right)\right\}}{1-\left\{1-\exp \left(-\alpha(x-\mu)^{2}\right)\right\}\left\{1+\lambda \exp \left(-\alpha(x-\mu)^{2}\right)\right\}},
\end{gathered}
$$

and

$$
H(x ; \alpha, \mu, \lambda)=-\log \left[1-\left\{1-\exp \left(-\alpha(x-\mu)^{2}\right)\right\}\left\{1+\lambda \exp \left(-\alpha(x-\mu)^{2}\right)\right\}\right] .
$$

Figure 2 shows the hazard rates of the TR distribution with different choices of parameters. The TR distribution has increasing behaviour for bathtub shaped hazard rates with different choices of parameters $\alpha, \lambda$ and the location parameter $\mu=0$.
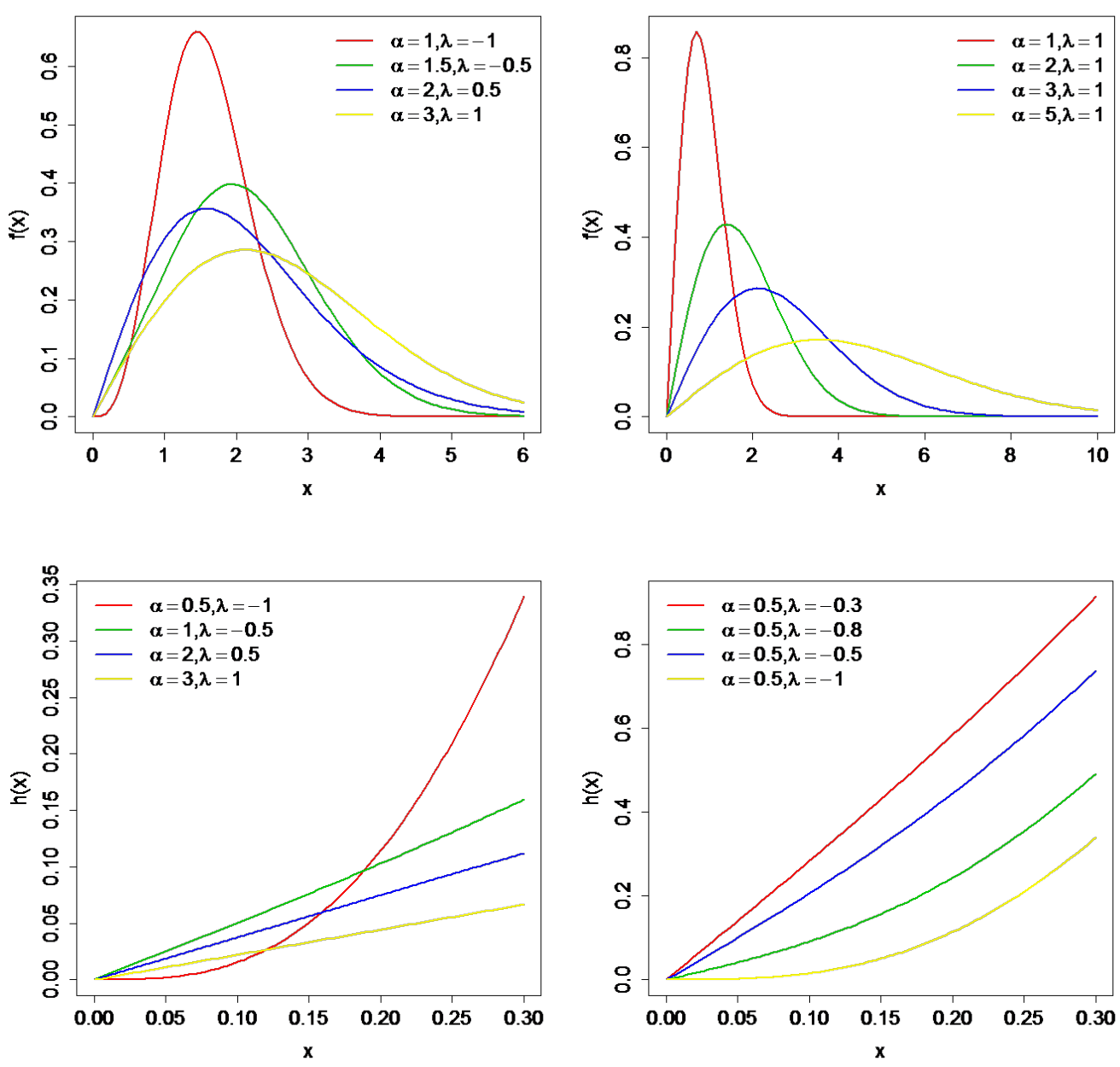

Fig. 2. Plots of the TR PDF \& HF for some parameter values.

\section{Moments and Quantiles}

This section presents the formulation of the $k^{t h}$ moment, incomplete moments, probability weighted moments, moment generating function and quantile function of the $\operatorname{TR}(x ; \alpha, \mu, \lambda)$ distribution. 
Theorem 1. If $X$ has the $T R(x ; \alpha, \mu, \lambda)$ with $|\lambda| \leq 1$, then the $k^{\text {th }}$ moment about $(X-\mu)$ is given as follows

$$
E(X-\mu)^{k}=\left\{(1-\lambda)+\lambda 2^{-k / 2}\right\} \alpha^{-k / 2} \Gamma\left(\frac{k}{2}+1\right) .
$$

Proof. By definition the $k^{\text {th }}$ moment of the TR distribution as follows

$$
E(X-\mu)^{k}=\int_{\mu}^{\infty} 2 \alpha(x-\mu)^{k+1} \exp \left(-\alpha(x-\mu)^{2}\right)\left\{1-\lambda+2 \lambda \exp \left(-\alpha(x-\mu)^{2}\right)\right\} d x,
$$

the above equation reduces to

$E(X-\mu)^{k}=(1-\lambda) 2 \alpha \int_{\mu}^{\infty}(x-\mu)^{k+1} \exp \left(-\alpha(x-\mu)^{2}\right) d x$

$$
+4 \alpha \lambda \int_{\mu}^{\infty}(x-\mu)^{k+1} \exp \left(-2 \alpha(x-\mu)^{2}\right) d x
$$

the above integral yields the $k^{\text {th }}$ moment

$$
E(X-\mu)^{k}=(1-\lambda) \alpha^{-k / 2} \Gamma\left(\frac{k}{2}+1\right)+\lambda(2 \alpha)^{-k / 2} \Gamma\left(\frac{k}{2}+1\right) .
$$

Corollary: The $k^{\text {th }}$ moment of the two parameter Rayleigh distribution can be obtained from equation (16) when the location parameter $\mu=0$ as

$$
E(X)^{k}=(1-\lambda) \alpha^{-k / 2} \Gamma\left(\frac{k}{2}+1\right)+\lambda(2 \alpha)^{-k / 2} \Gamma\left(\frac{k}{2}+1\right) .
$$

Theorem 2. If $X$ has the $T R(x ; \alpha, \mu, \lambda)$ distribution with $|\lambda| \leq 1$, then the $k^{\text {th }}$ incomplete moment about $(z-\mu)$ is given as follows

$$
\mu_{k}(z-\mu)=2^{-k / 2}\left\{(1-\lambda) \gamma\left(\frac{k}{2}+1, \alpha(z-\mu)^{2}\right)+\lambda \alpha^{-k / 2} \gamma\left(\frac{k}{2}+1,2 \alpha(z-\mu)^{2}\right)\right\} .
$$

Proof. By definition the $k^{\text {th }}$ incomplete moment can be defined as

$\mu_{k}(z-\mu)=(1-\lambda) 2 \alpha \int_{\mu}^{z}(x-\mu)^{k+1} \exp \left(-\alpha(x-\mu)^{2}\right) d x$

$$
+4 \alpha \lambda \int_{\mu}^{z}(x-\mu)^{k+1} \exp \left(-2 \alpha(x-\mu)^{2}\right) d x
$$

The above integral reduces to the $k^{\text {th }}$ incomplete moment as

$$
\mu_{k}(z-\mu)=(1-\lambda) 2^{-\mathrm{k} / 2 \gamma}\left(\frac{k}{2}+1, \alpha(z-\mu)^{2}\right)+\lambda(2 \alpha)^{-\mathrm{k} / 2} \gamma\left(\frac{k}{2}+1,2 \alpha(z-\mu)^{2}\right) .
$$

Theorem 3. If $X$ has the $T R(x ; \alpha, \mu, \lambda)$ with $|\lambda| \leq 1$, then the PWMs of $X$ say $\psi_{k, m}=E\left[(x-\mu)^{k} F(x)^{m}\right]$ is given as follows

$$
\psi_{k, m}=\sum_{i, j=0}^{\infty}\left(\begin{array}{c}
m \\
i
\end{array}\right)\left(\begin{array}{c}
m \\
j
\end{array}\right)(-1)^{i} \lambda^{j} \alpha^{-k / 2} \Gamma\left(\frac{k}{2}+1\right)\left\{\frac{(1-\lambda)}{(i+j+1)^{\frac{k}{2}+1}}+\frac{2 \lambda}{(i+j+2)^{\frac{k}{2}+1}}\right\} .
$$


Proof. The probability weighted moment of the TRD as follows

$$
\begin{aligned}
\psi_{k, m}=2 \alpha \int_{\mu}^{\infty}(x-\mu)^{k+1} \exp \left(-\alpha(x-\mu)^{2}\right) & \left\{1-\lambda+2 \lambda \exp \left(-\alpha(x-\mu)^{2}\right)\right\} \\
\times & \times\left\{1-\exp \left(-\alpha(x-\mu)^{2}\right)\right\}^{m}\left\{\left\{1+\lambda \exp \left(-\alpha(x-\mu)^{2}\right)\right\}\right\}^{m} d x,
\end{aligned}
$$

the above integral reduces to

$\psi_{k, m}=\varrho_{i, j} \int_{\mu}^{\infty}(x-\mu)^{k+1} \exp \left\{-(i+j+1) \alpha(x-\mu)^{2}\right\}\left\{1-\lambda+2 \lambda \exp \left(-\alpha(x-\mu)^{2}\right)\right\} d x$,

where

$$
\begin{gathered}
\varrho_{i, j}=2 \alpha \sum_{i, j=0}^{\infty}\left(\begin{array}{c}
m \\
i
\end{array}\right)\left(\begin{array}{c}
m \\
j
\end{array}\right)(-1)^{i} \lambda^{j} . \\
\psi_{k, m}=\varrho_{i, j}(1-\lambda) \int_{\mu}^{\infty}(x-\mu)^{k+1} \exp \left\{-(i+j+1) \alpha(x-\mu)^{2}\right\} d x \\
+2 \lambda \varrho_{i, j} \int_{\mu}^{\infty}(x-\mu)^{k+1} \exp \left\{-(i+j+2) \alpha(x-\mu)^{2}\right\} d x .
\end{gathered}
$$

Finally, we obtain

$$
\begin{aligned}
\psi_{k, m}=\sum_{i, j=0}^{\infty}\left(\begin{array}{c}
m \\
i
\end{array}\right)\left(\begin{array}{c}
m \\
j
\end{array}\right) \frac{(1-\lambda)(-1)^{i} \lambda^{j} \alpha^{-k / 2}}{(i+j+1)^{\frac{k}{2}+1}} \Gamma\left(\frac{k}{\alpha}+1\right) & +\sum_{i, j=0}^{\infty}\left(\begin{array}{c}
m \\
i
\end{array}\right)\left(\begin{array}{c}
m \\
j
\end{array}\right) \frac{2 \lambda(-1)^{i} \lambda^{j} \alpha^{-k / 2}}{(i+j+2)^{\frac{k}{2}+1}} \Gamma\left(\frac{k}{\alpha}+1\right) .
\end{aligned}
$$

Theorem 4. If $X$ is a random variable having the $T R(x ; \alpha, \mu, \lambda)$ with $|\lambda| \leq 1$, then the moment generating function of $X$ say $M_{X}(t)$ is given as follows

$$
M_{X}(t)=(1-\lambda) \sum_{m=0}^{\infty} \sum_{i=0}^{m}\left(\begin{array}{c}
m \\
i
\end{array}\right) \frac{t^{m} \mu^{m-i}}{m ! \alpha^{\frac{i}{2}}} \Gamma\left(\frac{i}{2}+1\right)+\lambda \sum_{m=0}^{\infty} \sum_{i=0}^{m}\left(\begin{array}{c}
m \\
i
\end{array}\right) \frac{t^{m} \mu^{m-i}}{m !(2 \alpha)^{\frac{i}{2}}} \Gamma\left(\frac{i}{2}+1\right) .
$$

Proof. By definition the mgf of the TR distribution as

$$
M_{X}(t)=(1-\lambda) 2 \alpha \int_{\mu}^{\infty}(x-\mu) \exp \left(t x-\alpha(x-\mu)^{2}\right) d x+4 \alpha \lambda \int_{\mu}^{\infty}(x-\mu) \exp \left(t x-2 \alpha(x-\mu)^{2}\right) d x,
$$

Using Taylor series expansions, the above integrals reduce to

$$
M_{X}(t)=(1-\lambda) \sum_{m=0}^{\infty} \frac{t^{m}}{m !} \int_{0}^{\infty}\left\{\sqrt{\left(\frac{w}{\alpha}\right)}+\mu\right\}^{m} \exp \{-w\} d w+\lambda \sum_{m=0}^{\infty} \frac{t^{m}}{m !} \int_{0}^{\infty}\left\{\sqrt{\left(\frac{w}{2 \alpha}\right)}+\mu\right\}^{m} \exp \{-w\} d w,
$$

Using the binomial expansion the above equation reduces to

$$
M_{X}(t)=(1-\lambda) \sum_{m=0}^{\infty} \sum_{i=0}^{m}\left(\begin{array}{c}
m \\
i
\end{array}\right) \frac{t^{m} \mu^{m-i}}{m ! \alpha^{\frac{i}{2}}} \Gamma\left(\frac{i}{2}+1\right)+\lambda \sum_{m=0}^{\infty} \sum_{i=0}^{m}\left(\begin{array}{c}
m \\
i
\end{array}\right) \frac{t^{m} \mu^{m-i}}{m !(2 \alpha)^{\frac{i}{2}}} \Gamma\left(\frac{i}{2}+1\right) .
$$




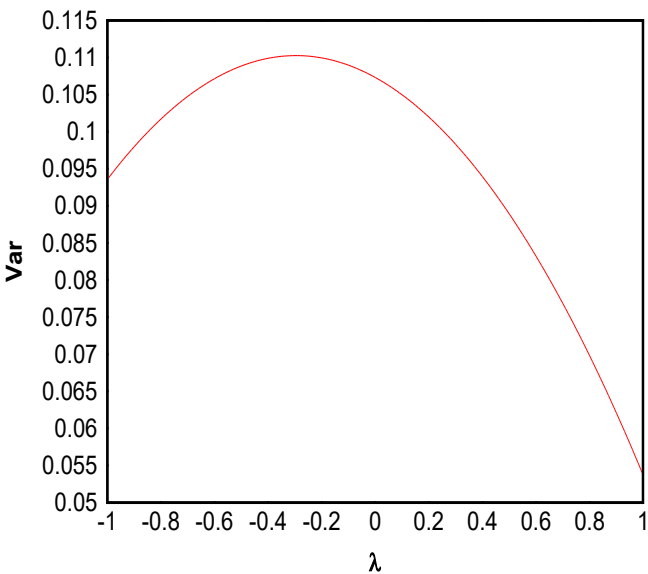

(a) Variance

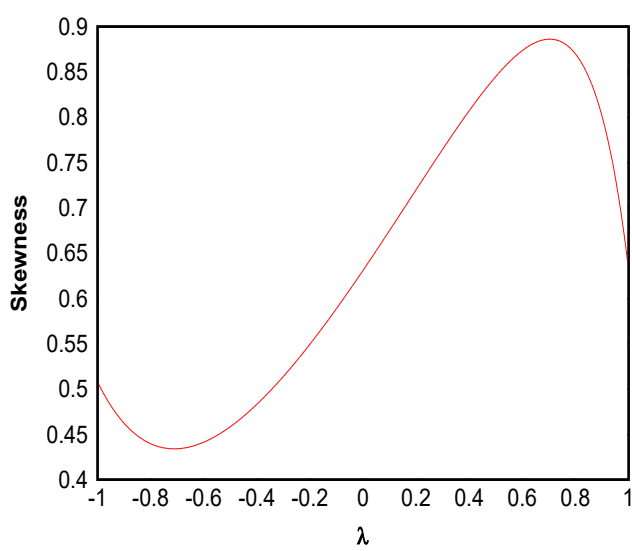

(c) Skewness

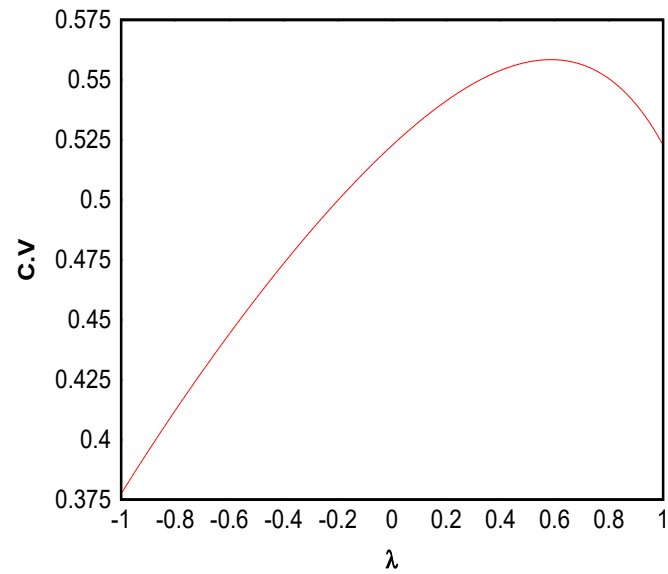

(b) Coefficient of Variation

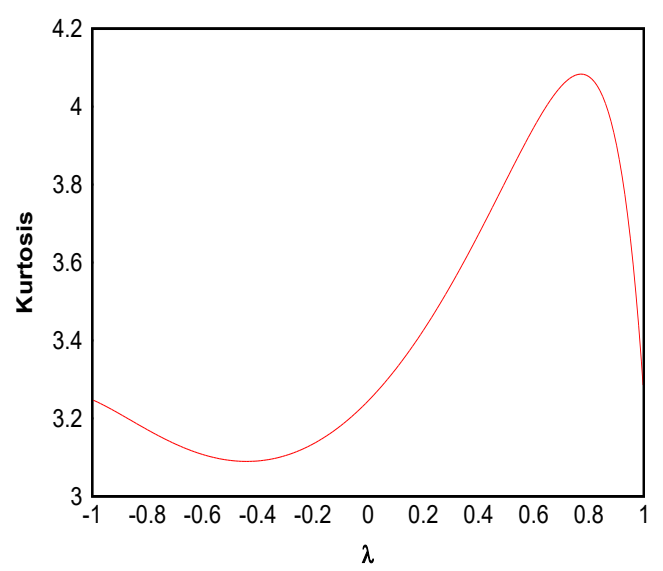

(d) Kurtosis

Fig. 3. Plots of the Variance, CV, Skewness and kurtosis of the TR distribution

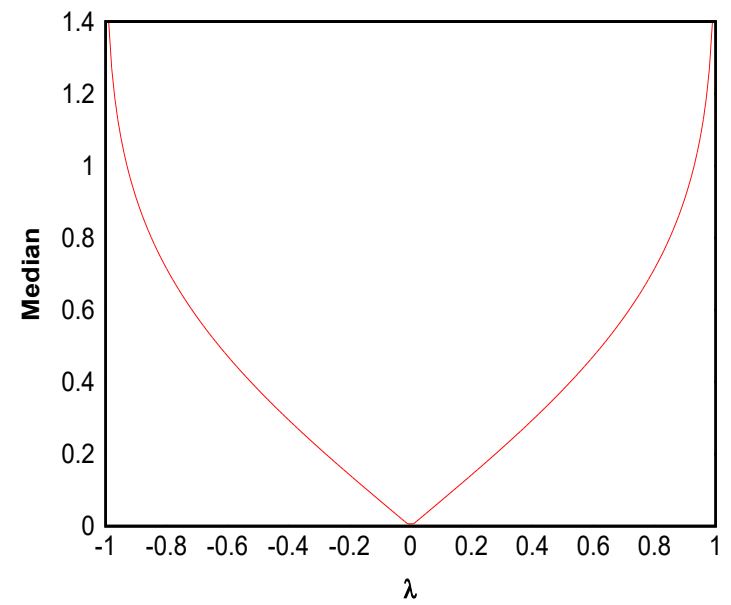

Fig. 4. Plot of the Median life of the TR distribution 
The quantile $F^{-1}(u)$ of the $\operatorname{TR}(x ; \alpha, \mu, \lambda)$ is the real solution of the following equation

$$
F^{-1}(u)=\mu+\sqrt{\frac{-1}{\alpha} \ln \left\{1-\frac{(1+\lambda)-\sqrt{(1+\lambda)^{2}-4 \lambda u}}{2 \lambda}\right\}}
$$

By substituting $u=0.5$ in (21) we obtain the median of $T R(x ; \alpha, \mu, \lambda)$ as follows

$$
F^{-1}(0.5)=\mu+\sqrt{\frac{-1}{\alpha} \ln \left\{1-\frac{(1+\lambda)-\sqrt{1+\lambda^{2}}}{2 \lambda}\right\}}
$$

Figure 3 displays the variance, coefficient of variation, coefficient of skewness and coefficient of kurtosis as a function of the parameter $\lambda$ and $\alpha=1$ by using equation (17). Figure 4 shows the median lifetime as a function of the parameter $\lambda$ and $\alpha=1$.

\section{Entropy and Mean Deviation}

The entropy of a random variable $\mathrm{X}$ with density $f(x)$ is a measure of the uncertainty in the data. A large value of entropy indicates the greater uncertainty. The Rényi entropy [21] is defined as

$$
I_{R}(\rho)=\frac{1}{1-\rho} \log \left\{\int f(x)^{\rho} d x\right\}
$$

where $\rho>0$ and $\rho \neq 1$. The integral in $I_{R}(\rho)$ of the $\operatorname{TR}(x ; \alpha, \mu, \lambda)$ can be defined as

$$
\int_{\mu}^{\infty} f(x)^{\rho} d x=\int_{\mu}^{\infty}(2 \alpha)^{\rho}(x-\mu)^{\rho} \exp \left(-\alpha \rho(x-\mu)^{2}\right)\left\{1-\lambda+2 \lambda \exp \left(-\alpha(x-\mu)^{2}\right)\right\}^{\rho} d x,
$$

the above integral reduces to

$$
\begin{aligned}
= & (1-\lambda)^{\rho} \sum_{i=0}^{\infty}\left(\begin{array}{c}
\rho \\
i
\end{array}\right)\left(\frac{2 \lambda}{1-\lambda}\right)^{i} \int_{\mu}^{\infty}(2 \alpha)^{\rho}(x-\mu)^{\rho} \exp \left(-\alpha(\rho+i)(x-\mu)^{2}\right) d x, \\
& \int_{\mu}^{\infty} f(x)^{\rho} d x=(1-\lambda)^{\rho} \sum_{i=0}^{\infty}\left(\begin{array}{c}
\rho \\
i
\end{array}\right)\left(\frac{2 \lambda}{1-\lambda}\right)^{i} \frac{\alpha^{\frac{\rho}{2}} 2^{\rho-1}}{(\rho+i)^{\frac{\rho}{2}}} \Gamma\left(\frac{\rho}{2}\right) .
\end{aligned}
$$

Finally we obtain the Rényi entropy as

$$
I_{R}(\rho)=-\log (2)+\frac{\rho}{2(1-\rho)} \log (\alpha)+\frac{\rho}{1-\rho} \log (1-\lambda)+\frac{1}{1-\rho} \log \left\{\sum_{i=0}^{\infty}\left(\begin{array}{c}
\rho \\
i
\end{array}\right)\left(\frac{2 \lambda}{1-\lambda}\right)^{i}(\rho+i)^{-\frac{\rho}{2}} \Gamma\left(\frac{\rho}{2}\right)\right\}
$$

The $\beta$ entropy was introduced by Havrda and Charvat [9] and is defined as

$$
I_{H}(\beta)=\frac{1}{\beta-1}\left\{1-\int_{0}^{\infty} f(x)^{\beta} d x\right\},
$$

where $\beta>0$ and $\beta \neq 1$. The integral in $I_{H}(\beta)$ of the TR distribution can be defined as 


$$
\int_{\mu}^{\infty} f(x)^{\beta} d t=\int_{\mu}^{\infty}(2 \alpha)^{\beta}(x-\mu)^{\beta} \exp \left(-\alpha \beta(x-\mu)^{2}\right)\left\{1-\lambda+2 \lambda \exp \left(-\alpha(x-\mu)^{2}\right)\right\}^{\beta} d x
$$

using (22) and (24), we obtain the expression of the $\beta$ entropy as

$$
I_{H}(\beta)=\frac{1}{\beta-1}\left\{1-(1-\lambda)^{\beta} \sum_{i=0}^{\infty}\left(\begin{array}{l}
\beta \\
i
\end{array}\right)\left(\frac{2 \lambda}{1-\lambda}\right)^{i} \frac{\alpha^{\frac{\beta}{2}} 2^{\beta-1}}{(\rho+i)^{\frac{\beta}{2}}} \Gamma\left(\frac{\beta}{2}\right)\right\} .
$$

The measure of dispersion in a population is measured by the totality of deviations from the mean and median. If $X$ has the $T R(x ; \alpha, \mu, \lambda)$, then we can derive the mean deviation about the mean $\mu=E(x)$ and about the median $\mathrm{M}$ by following equations

$$
\delta_{1}=2 \mu F(\mu)-2 \psi(\mu) \quad \text { and } \quad \delta_{2}=\mu-2 \psi(M)
$$

The mean $\mu=E(x)$ is obtained from (17) with $k=1$ and the median $M$ is obtained from the solution of the non-linear equation (21), where $\psi(q)$ can be derived from (11)

$$
\psi(q)=(1-\lambda) 2^{-1 / 2} \gamma\left(\frac{1}{2}+1, \alpha(z-\mu)^{2}\right)+\lambda(2 \alpha)^{-1 / 2} \gamma\left(\frac{1}{2}+1,2 \alpha(z-\mu)^{2}\right),
$$

where $\gamma(\beta, t)=\int_{0}^{t} w^{\beta-1} e^{-w} d w$ is the incomplete gamma function. Hence, the deviation measure in (25) can be obtained from (26). The quantity $\psi(q)$ can also be used to determine Bonferroni and Lorenz curves and they are given by

$$
\begin{aligned}
& B(P)=\frac{1}{P \mu}\left\{(1-\lambda) 2^{-1 / 2} \gamma\left(\frac{1}{2}+1, \alpha(z-\mu)^{2}\right)+\lambda(2 \alpha)^{-1 / 2} \gamma\left(\frac{1}{2}+1,2 \alpha(z-\mu)^{2}\right)\right\} . \\
& L(P)=\frac{1}{\mu}\left\{(1-\lambda) 2^{-1 / 2} \gamma\left(\frac{1}{2}+1, \alpha(z-\mu)^{2}\right)+\lambda(2 \alpha)^{-1 / 2} \gamma\left(\frac{1}{2}+1,2 \alpha(z-\mu)^{2}\right)\right\} .
\end{aligned}
$$

where $q=Q(P)$ is calculated from (12) for a given probability $P$.

\section{Order Statistics}

Let $x_{1}, x_{2}, \ldots, x_{n}$ be independently and identically distributed ordered random variables from the $\operatorname{TR}(x ; \alpha, \mu, \lambda)$ distribution having the pdf of $\mathrm{rth}$ order statistics is given by

$$
f_{r: n}(x)=\frac{1}{B(r, n-r+1)} \sum_{m=0}^{n-r}\left(\begin{array}{c}
n-r \\
m
\end{array}\right)(-1)^{m}(F(x))^{r+m-1} f(x),
$$

Substituting (11) and (12) in (27) we obtain

$$
\begin{aligned}
f_{r: n}(x)=n\left(\begin{array}{l}
n-1 \\
r-1
\end{array}\right) \sum_{m=0}^{n-r} \sum_{i, j=0}^{\infty}\left(\begin{array}{c}
n-r \\
m
\end{array}\right) & \left.\begin{array}{c}
r+m-1 \\
i
\end{array}\right)\left(\begin{array}{c}
r+m-1 \\
j
\end{array}\right)(-1)^{m+j} 2 \alpha(x-\mu) \\
& \times \exp \left(-\alpha(i+j+1)(x-\mu)^{2}\right)\left\{1-\lambda+2 \lambda \exp \left(-\alpha(x-\mu)^{2}\right)\right\} .
\end{aligned}
$$


By using equation (19), we obtain the expression for the moments of order statistics of the three parameter transmuted Rayleigh distribution can be defined as

$$
E\left(X_{r: n}-\mu\right)^{K}=n\left(\begin{array}{c}
n-1 \\
r-1
\end{array}\right) \sum_{m=0}^{n-r} \sum_{i, j=0}^{\infty} v_{i, j, m} \psi_{k, r+m-1}
$$

where

$$
v_{i, j, m}=\left(\begin{array}{c}
n-r \\
m
\end{array}\right)\left(\begin{array}{c}
r+m-1 \\
i
\end{array}\right)\left(\begin{array}{c}
r+m-1 \\
j
\end{array}\right)(-1)^{m+j} .
$$

where $\psi_{k, r+m-1}$ is given in Section 3, Theorem 3 .

The measure of skewness and kurtosis of the distribution of $k^{\text {th }}$ moment of $r$ th order statistics can be formulated by using equation (28).

\section{Parameter Estimation}

Suppose $x_{1}, x_{2}, \ldots, x_{n}$ be a random samples consisting of $n$ observations from the three parameter transmuted Rayleigh distribution. Let $\Theta=(\alpha, \mu, \lambda)^{\mathrm{T}}$ be the vector of the parameters. Then the log-likelihood function for the vector of parameters $\Theta$ can be expressed as

$$
\mathcal{L}(\Theta)=n \ln (2)+n \ln \alpha+\sum_{i=1}^{n} \ln \left(x_{i}-\mu\right)-\alpha \sum_{i=1}^{n}\left(x_{i}-\mu\right)^{2}+\sum_{i=1}^{n} \ln \left\{1-\lambda+2 \lambda \exp \left\{-\alpha\left(x_{i}-\mu\right)^{2}\right\}\right\}
$$

The first order partial derivative with respect to $\alpha, \mu$ and $\lambda$ then equating it to zero, we obtain the component of the score vector $U(\Theta)$ is given by

$$
\begin{aligned}
& \frac{\partial \mathcal{L}(\Theta)}{\partial \alpha}=\frac{n}{\alpha}-\sum_{i=1}^{n}\left(x_{i}-\mu\right)^{2}-\sum_{i=1}^{n} \frac{2 \lambda \exp \left\{-\alpha\left(x_{i}-\mu\right)^{2}\right\}\left(x_{i}-\mu\right)^{2}}{\left\{1-\lambda+2 \lambda \exp \left\{-\alpha\left(x_{i}-\mu\right)^{2}\right\}\right\}} \\
& \frac{\partial \mathcal{L}(\Theta)}{\partial \mu}=-\sum_{i=1}^{n} \frac{1}{\left(x_{i}-\mu\right)}+2 \alpha \sum_{i=1}^{n}\left(x_{i}-\mu\right)+\sum_{i=1}^{n} \frac{4 \alpha \lambda \exp \left\{-\alpha\left(x_{i}-\mu\right)^{2}\right\}\left(x_{i}-\mu\right)}{\left\{1-\lambda+2 \lambda \exp \left\{-\alpha\left(x_{i}-\mu\right)^{2}\right\}\right\}} \\
& \frac{\partial \mathcal{L}(\Theta)}{\partial \lambda}=\sum_{i=1}^{n} \frac{2 \exp \left\{-\alpha\left(x_{i}-\mu\right)^{2}\right\}-1}{\left\{1-\lambda+2 \lambda \exp \left\{-\alpha\left(x_{i}-\mu\right)^{2}\right\}\right\}}
\end{aligned}
$$

respectively. The maximum likelihood estimates for the parameter vector $\Theta=(\alpha, \mu, \lambda)^{\mathrm{T}}$ can be obtained by solving the simultaneous equation of the first order partial derivatives. For testing of hypothesis and confidence interval for the parameter vector $\Theta=(\alpha, \mu, \lambda)^{\mathrm{T}}$ we considered the multivariate normal distribution with the variance covariance matrix and its inverse of the expected information matrix are given by

and

$$
\left(\begin{array}{l}
\hat{\alpha} \\
\hat{\mu} \\
\hat{\lambda}
\end{array}\right) \sim N\left[\left(\begin{array}{l}
\alpha \\
\mu \\
\lambda
\end{array}\right),\left(\begin{array}{lll}
\hat{V}_{11} & \hat{V}_{12} & \hat{V}_{13} \\
\hat{V}_{21} & \widehat{V}_{22} & \widehat{V}_{23} \\
\hat{V}_{31} & \widehat{V}_{32} & \widehat{V}_{33}
\end{array}\right)\right]
$$




$$
V^{-1}=-E\left[\begin{array}{ccc}
\frac{\partial^{2} \mathcal{L}(\Theta)}{\partial \alpha^{2}} & \frac{\partial^{2} \mathcal{L}(\Theta)}{\partial \alpha \partial \mu} & \frac{\partial^{2} \mathcal{L}(\Theta)}{\partial \alpha \partial \lambda} \\
\frac{\partial^{2} \mathcal{L}(\Theta)}{\partial \alpha \partial \mu} & \frac{\partial^{2} \mathcal{L}(\Theta)}{\partial \mu^{2}} & \frac{\partial^{2} \mathcal{L}(\Theta)}{\partial \mu \partial \lambda} \\
\frac{\partial^{2} \mathcal{L}(\Theta)}{\partial \alpha \partial \lambda} & \frac{\partial^{2} \mathcal{L}(\Theta)}{\partial \mu \partial \lambda} & \frac{\partial^{2} \mathcal{L}(\Theta)}{\partial \lambda^{2}}
\end{array}\right]
$$

The $3 \times 3$ unit observed information matrix, whose elements are given by

$$
\begin{aligned}
& \frac{\partial^{2} \mathcal{L}(\Theta)}{\partial \alpha^{2}}=-\frac{n}{\alpha^{2}}+\sum_{i=1}^{n} \frac{2 \lambda \exp \left\{-\alpha\left(x_{i}-\mu\right)^{2}\right\}\left(x_{i}-\mu\right)^{4}}{\left\{1-\lambda+2 \lambda \exp \left\{-\alpha\left(x_{i}-\mu\right)^{2}\right\}\right\}^{2}}, \\
& \frac{\partial^{2} \mathcal{L}(\Theta)}{\partial \mu^{2}}=-2 \alpha-\sum_{i=1}^{n} \frac{1}{\left(x_{i}-\mu\right)^{2}} \\
& +\sum_{i=1}^{n} \frac{4 \alpha \lambda \exp \left\{-\alpha\left(x_{i}-\mu\right)^{2}\right\}\left\{(1-\lambda)\left(2 \alpha\left(x_{i}-\mu\right)^{2}-1\right)-2 \lambda \exp \left\{-\alpha\left(x_{i}-\mu\right)^{2}\right\}\right\}}{\left\{1-\lambda+2 \lambda \exp \left\{-\alpha\left(x_{i}-\mu\right)^{2}\right\}\right\}^{2}}, \\
& \frac{\partial^{2} \mathcal{L}(\Theta)}{\partial \lambda^{2}}=-\sum_{i=1}^{n} \frac{\left\{2 \exp \left\{-\alpha\left(x_{i}-\mu\right)^{2}\right\}-1\right\}^{2}}{\left\{1-\lambda+2 \lambda \exp \left\{-\alpha\left(x_{i}-\mu\right)^{2}\right\}\right\}^{2}} \text {. } \\
& \frac{\partial^{2} \mathcal{L}(\Theta)}{\partial \alpha \partial \mu}=2 \sum_{i=1}^{n}\left(x_{i}-\mu\right) \\
& -\sum_{i=1}^{n} \frac{4 \alpha \lambda\left(x_{i}-\mu\right) \exp \left\{-\alpha\left(x_{i}-\mu\right)^{2}\right\}\left\{(\lambda-1)+\alpha(1-\lambda)\left(x_{i}-\mu\right)^{2}-2 \lambda \exp \left\{-\alpha\left(x_{i}-\mu\right)^{2}\right\}\right\}}{\left\{1-\lambda+2 \lambda \exp \left\{-\alpha\left(x_{i}-\mu\right)^{2}\right\}\right\}^{2}}, \\
& \frac{\partial^{2} \mathcal{L}(\Theta)}{\partial \mu \partial \lambda}=\sum_{i=1}^{n} \frac{8 \alpha^{2} \lambda(1-\lambda)\left(x_{i}-\mu\right)^{2} \exp \left\{-2 \alpha\left(x_{i}-\mu\right)^{2}\right\}}{\left\{1-\lambda+2 \lambda \exp \left\{-\alpha\left(x_{i}-\mu\right)^{2}\right\}\right\}^{2}} \\
& \frac{\partial^{2} \mathcal{L}(\Theta)}{\partial \alpha \partial \lambda}=-\sum_{i=1}^{n} \frac{2 \exp \left\{-\alpha\left(x_{i}-\mu\right)^{2}\right\}\left(x_{i}-\mu\right)^{2}}{\left\{1-\lambda+2 \lambda \exp \left\{-\alpha\left(x_{i}-\mu\right)^{2}\right\}\right\}^{2}}
\end{aligned}
$$

The multivariate normal distribution can be used to obtain as an approximate $100(1-\gamma) \%$ confidence intervals for the parameters $\alpha, \mu$ and $\lambda$ can be determined as

$$
\hat{\alpha} \pm Z_{\frac{\gamma}{2}} \sqrt{\widehat{V}_{11}}, \quad \hat{\mu} \pm Z_{\frac{\gamma}{2}} \sqrt{\widehat{V}_{22}}, \quad \hat{\lambda} \pm Z_{\frac{\gamma}{2}} \sqrt{\widehat{V}_{33}},
$$

where $Z_{\frac{\gamma}{2}}$ is the upper $\alpha$ th percentile of the standard normal distribution.

\section{Simulation}

In this section we evaluate the performance of the MLEs for the three parameter transmuted Rayleigh distribution. Let $U$ denote the uniform random variable over the interval $[0,1]$. We generate the random variable of the subject distribution by using equation (21) and the simulation were carried out by using the statistical software package R. Using the Monte Carlo simulation we generate the random samples of size 
$n=25,50,75,100,200,300,400,500$ from the three parameter transmuted Rayleigh distribution and the BFGS method has been used to minimize the total log likelihood function. We consider the fixed choice of parameter values $\alpha=1, \mu=0.5, \lambda=0.5$. The simulation process is repeated for 1000 times and the results were displayed in Table 1. Simulation study has been performed in order to evaluate the mean estimates, standard error (S.E), bias and mean square error (MSE). These results of simulation suggest that as the sample size $n$ increases the method of MLEs provide the better estimates. Figure 5 illustrates the transmuted Rayleigh density with histogram for simulated data set. The plot in Figure 5 confirms that the distribution has positively skewed behaviour of the histogram.

Table 1: Mean, standard Error, Bias and MSE of the 3P-TR distribution

\begin{tabular}{|c|c|c|c|c|c|}
\hline$n$ & Parameter & Mean & S.E & Bias & $M S E$ \\
\hline \multirow{3}{*}{25} & $\alpha$ & 1.6269 & 0.7228 & 0.6269 & 0.9154 \\
\hline & $\mu$ & 0.4536 & 0.0752 & -0.0464 & 0.0078 \\
\hline & $\lambda$ & 0.0100 & 0.8870 & -0.4900 & 1.0268 \\
\hline \multirow{3}{*}{50} & $\alpha$ & 1.2563 & 0.2948 & 0.2563 & 0.1525 \\
\hline & $\mu$ & 0.6120 & 0.0589 & 0.1120 & 0.0160 \\
\hline & $\lambda$ & 0.0194 & 0.4122 & -0.4806 & 0.4008 \\
\hline \multirow{3}{*}{75} & $\alpha$ & 1.1185 & 0.3721 & 0.1185 & 0.1525 \\
\hline & $\mu$ & 0.5438 & 0.0383 & 0.0438 & 0.0033 \\
\hline & $\lambda$ & 0.5617 & 0.4330 & 0.0617 & 0.1912 \\
\hline \multirow{3}{*}{100} & $\alpha$ & 1.0759 & 0.2988 & 0.0759 & 0.0950 \\
\hline & $\mu$ & 0.4960 & 0.0377 & -0.0040 & 0.0014 \\
\hline & $\lambda$ & 0.4006 & 0.4084 & -0.0994 & 0.1766 \\
\hline \multirow{3}{*}{200} & $\alpha$ & 0.9750 & 0.2645 & -0.0250 & 0.0705 \\
\hline & $\mu$ & 0.4983 & 0.0273 & -0.0017 & 0.0007 \\
\hline & $\lambda$ & 0.5151 & 0.3780 & 0.0151 & 0.1431 \\
\hline \multirow{3}{*}{300} & $\alpha$ & 1.1567 & 0.2204 & 0.1567 & 0.0731 \\
\hline & $\mu$ & 0.5288 & 0.0197 & 0.0288 & 0.0012 \\
\hline & $\lambda$ & 0.4685 & 0.2734 & -0.0315 & 0.0757 \\
\hline \multirow{3}{*}{400} & $\alpha$ & 1.0331 & 0.1291 & 0.0331 & 0.0177 \\
\hline & $\mu$ & 0.5193 & 0.0189 & 0.0193 & 0.0007 \\
\hline & $\lambda$ & 0.5826 & 0.1526 & 0.0826 & 0.0301 \\
\hline \multirow{3}{*}{500} & $\alpha$ & 0.9350 & 0.1564 & -0.0650 & 0.0286 \\
\hline & $\mu$ & 0.4941 & 0.0164 & -0.0059 & 0.0003 \\
\hline & $\lambda$ & 0.5939 & 0.2174 & 0.0939 & 0.0560 \\
\hline
\end{tabular}




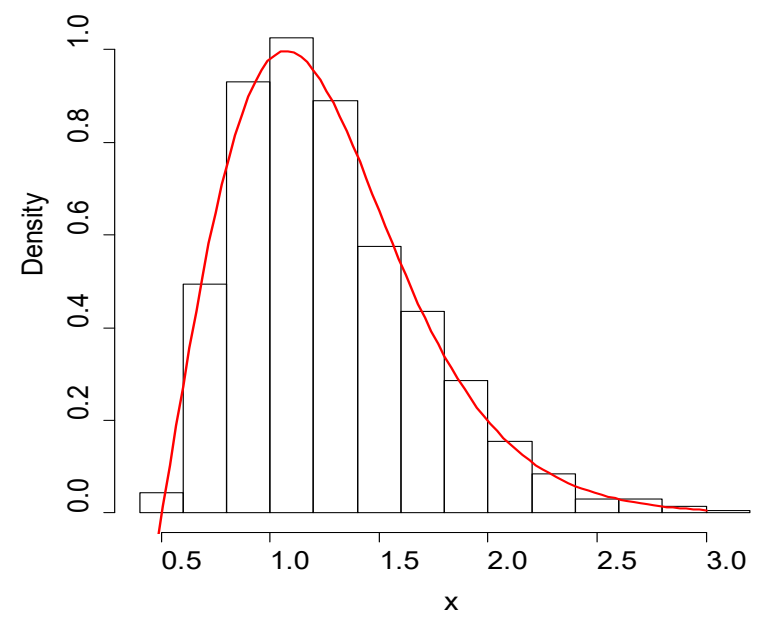

Fig. 5. Plot of the TR density for simulated data set: $\alpha=1, \mu=0.5, \lambda=0.5$.

\section{Application}

In this section we consider the data set represents the life of fatigue fracture of Kevlar 373/epoxy that are subject to constant pressure at the $90 \%$ stress level until all components had failed. The data set consist of 76 observations with the exact times of failures. The data have been obtained from Yolanda et al. [28] and is given below $0.0251,0.0886,0.0891,0.2501,0.3113,0.3451,0.4763,0.5650,0.5671,0.6566,0.6748,0.6751,0.6753,0.7696,0.83$ $75,0.8391,0.8425,0.8645,0.8851,0.9113,0.9120,0.9836,1.0483,1.0596,1.0773,1.1733,1.2570,1.2766,1.2985,1$. $3211,1.3503,1.3551,1.4595,1.4880,1.5728,1.5733,1.7083,1.7263,1.7460,1.7630,1.7746,1.8275,1.8375,1.8503$ ,1.8808,1.8878,1.8881,1.9316,1.9558,2.0048,2.0408,2.0903,2.1093,2.1330,2.2100,2.2460,2.2878,2.3203,2.34 $70,2.3513,2.4951,2.5260,2.9911,3.0256,3.2678,3.4045,3.4846,3.7433,3.7455,3.9143,4.8073,5.4005,5.4435$, $5.5295,6.5541,9.0960$.

We compare the three parameter transmuted Rayleigh (3P-TR), two parameter Rayleigh (2P-R), Weighted Rayleigh (WR) and Rayleigh (R) distributions fitted to the fatigue fracture data. The required computation were performed by the BFGS method to minimize the total log likelihood function by using the statistical software package $\mathrm{R}$ [22]. Table 2 displays the maximum likelihood estimates (MLEs) with their corresponding standard errors of the parameters and the values of the AIC (Akaike information criteria), CAIC (Consistent Akaikes Information Criterion), BIC (Bayesian information criterion) for four lifetime distributions.

The results from Table 2 indicate that the 3P-TR distribution has the lowest AIC, BIC and CAIC values than the 2P-R, WR and R distributions and therefore it could be chosen as the best model. We also perform the graphical goodness of fit in Figure 6, Plots of the histogram of the data with the fitted two different lifetime distributions. Figure 7 gives the empirical and fitted cumulative distribution functions of the three parameter transmuted Rayleigh (3P-TR) and two parameter Rayleigh (2P-R) distributions. We conclude from these plots that the 3P-TR distribution provides better fit than the 2P-R, WR and $\mathrm{R}$ distributions. Therefore the proposed model offers an attractive alternative to the $2 \mathrm{P}-\mathrm{R}$, WR and $\mathrm{R}$ distributions. Table 2 also suggests that the 3PTR distribution provides the better fit for an application of the fatigue fracture data. 


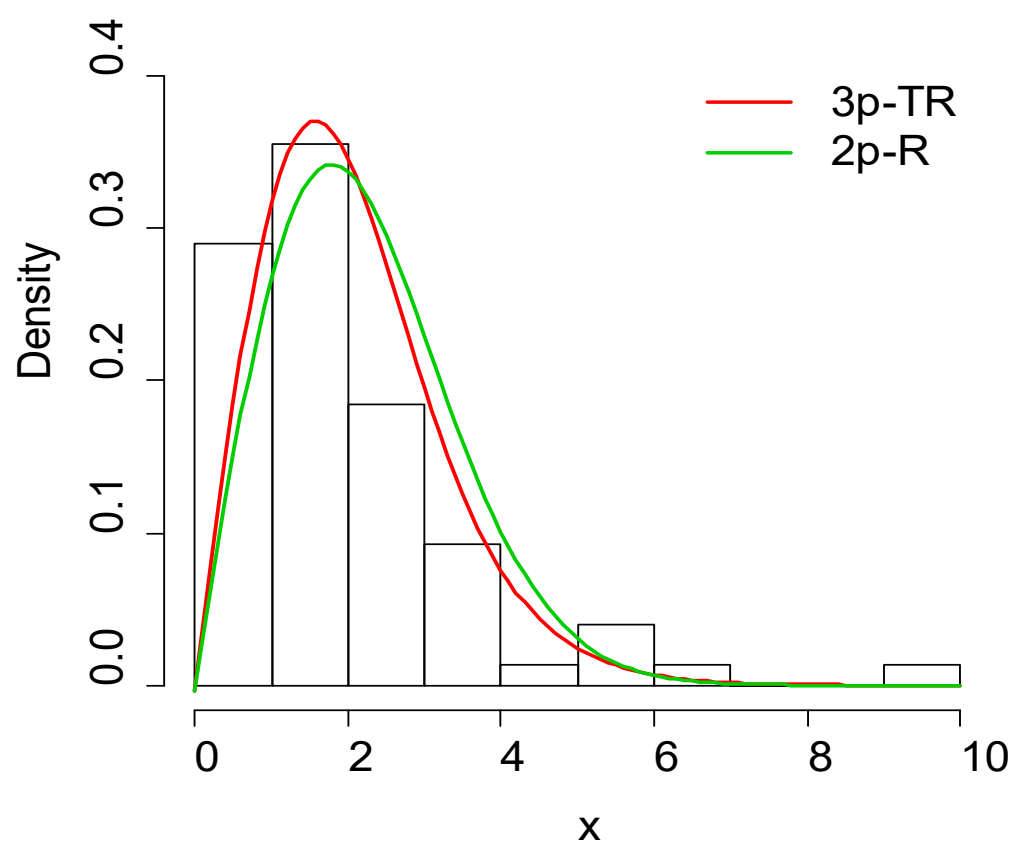

Fig. 6. Estimated densities of the fatigue fracture data.

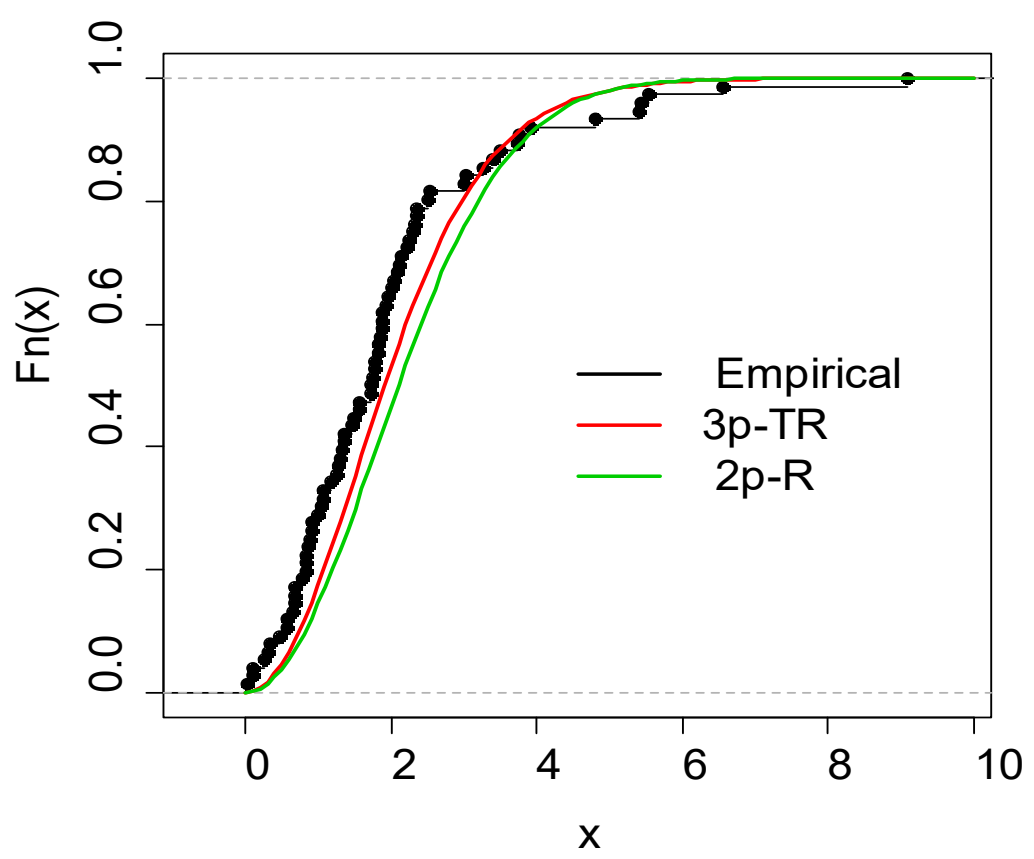

Fig. 7. Estimated cdf functions for the fatigue fracture data. 
Table 2: MLEs of the unknown Parameters for the fatigue fracture data with their corresponding standard errors in parenthesis and the goodness of fit measures, AIC, BIC, CAIC

\begin{tabular}{l|ccc|ccc}
\hline \multirow{2}{*}{ Distribution } & \multicolumn{3}{|c|}{ Parameter Estimates } & \multirow{2}{*}{ AIC } & BIC & CAIC \\
\cline { 2 - 4 } & $\hat{\alpha}$ & $\hat{\mu}$ & $\hat{\lambda}$ & & & \\
\hline 3P-TR & 0.1160 & 0.0099 & 0.7035 & 267.74 & 274.73 & 268.07 \\
& $(0.0175)$ & $(0.0228)$ & $(0.1495)$ & & & \\
2P-R & 0.1591 & 0.0012 & - & 278.63 & 283.30 & 278.80 \\
& $(0.0183)$ & $(0.0228)$ & & & & \\
WR & $6.8 \mathrm{e}+4$ & - & 0.1591 & 278.62 & 283.29 & 278.79 \\
& $(1.1 \mathrm{e}+4)$ & & $(0.3001)$ & & & \\
$\mathrm{R}$ & 0.1591 & - & - & 276.63 & 278.97 & 276.69 \\
& $(0.0182)$ & & & & & \\
\hline
\end{tabular}

Using the maximum likelihood estimates of the unknown parameters for fatigue fracture data with testing and the approximately $95 \%$ two sided confidence interval for the parameters $\alpha, \mu$ and $\lambda$ of the 3P-TR distribution displayed in Table 3.

Table 3: Estimated Parameters of the 3P-TR distribution for fatigue fracture data

\begin{tabular}{c|ccccc|c}
\hline Parameter & ML & Standard & t-Stat & \multicolumn{2}{c}{$95 \%$ Confidence Interval } & P-Value \\
& Estimates & Error & & Lower & Upper & \\
\hline$\hat{\alpha}$ & 0.1160 & 0.0175 & 6.6285 & 0.0817 & 0.1503 & $4.5 \mathrm{E}-09$ \\
$\hat{\mu}$ & 0.0099 & 0.0228 & 0.4342 & -0.0347 & 0.0545 & 0.6653 \\
$\hat{\lambda}$ & 0.7035 & 0.1495 & 4.7056 & 0.4104 & 0.9965 & $1.13 \mathrm{E}-05$ \\
\hline
\end{tabular}

\section{Conclusion}

We introduce a new distribution, called the 3P-TR distribution and studied its various structural properties. We also introduce the new class of weighted Rayleigh distribution and formulated its $k^{\text {th }}$ moment which has closed form expression. We obtain the analytical shapes of density and hazard functions of the 3P-TR distribution. We derive the expressions for the moments, incomplete moments, probability weighted moments, moment generating function, quantile function, mean deviations, Bonferroni and Lorenz curves, Renyi and Beta entropies and the moments of order statistics. Maximum likelihood estimation is discussed with asymptotic log likelihood inference including confidence intervals. The importance and flexibility of the transmuting parameter in the 3P-TR distribution is accessed by simulation. The usefulness of the proposed model is illustrated in an application to fatigue fracture data and conclude that the three parameter transmuted Rayleigh distribution provides better fit than the other three models.

\section{References}

[1] Aarset, M.V. (1987), "How to identify a bathtub shaped hazard rate?",IEEE Transactions on Reliability, vol. 36, 106 - 108.

[2] A. Azzalini, (1985). A class of distributions which includes the normal ones, Scand. J. Stat. 12, 171-178.

[3] Aryal G R., Tsokos C. P., (2009). On the transmuted extreme value distribution with applications. Nonlinear Analysis: Theory, Methods and applications, Vol. 71, 2009, 1401-1407.

[4] Aryal G R., Tsokos C. P., (2011). Transmuted Weibull distribution: A Generalization of the Weibull Probability Distribution. European Journal of Pure and Applied Mathematics, Vol. 4, No. 2, 89-102.

[5] Dey, S. (2009), "Comparison of Bayes Estimators of the parameter and reliability function for Rayleigh distribution under different loss functions", Malaysian Journal of Mathematical Sciences, Vol. 3, 247-264.

[6] Dey, S. and Das, M.K. (2007), “A Note on Prediction Interval for a Rayleigh Distribution: Bayesian Approach”, American Journal of Mathematical and Management Science, Vol. 27 (1\&2), 43 - 48. 
[7] Elbatal. I., Diab. L.S., Abdul Alim. N. A., (2013). Transmuted Generalized Linear Exponential Distribution, International Journal of Computer Applications, Vol. 83 - No. 17, Dec. 2013, 29-37.

[8] Gupta. R. D and Kundu. D, (2009). A new class of weighted exponential distributions, Statistics: A Journal of Theoretical and Applied Statistics, Vol. 43:6, 621-634.

[9] Havrda, J. and Charvat, F., (1967). Quantification method in classification processes: concept of structural $\alpha$-entropy. Kybernetika, Vol. 3, 30-35.

[10] Johnson, N.L., Kotz, S. and Balakrishnan, N., (1995). Continuous Univariate Distribution Vol. 1, 2nd Edition, John Wiley \& Sons, New York.

[11] Khan M. Shuaib, King Robert,. (2013a). Transmuted Modified Weibull Distribution: A Generalization of the Modified Weibull Probability Distribution, European Journal of Pure And Applied Mathematics, Vol. 6, No. 1, 66-88.

[12] Khan M. Shuaib, King Robert,. (2013b). Transmuted Generalized Inverse Weibull Distribution, Journal of Applied Statistical Sciences, Vol. 20, No. 3, 15-32.

[13] Khan M. Shuaib and King Robert. (2014). A New Class of Transmuted Inverse Weibull Distribution for Reliability Analysis, American Journal of Mathematical and Management Sciences, Vol. 33:4, 261-286.

[14] Khan, M. Shuaib, King Robert. (2015). Transmuted Modified Inverse Rayleigh Distribution, Austrian Journal of Statistics, Vol 44, No. 3, 2015, pp. 17-29.

[15] Khan, M. Shuaib, King Robert, Hudson Irene., (2015). A new three parameter transmuted Chen lifetime distribution with application, Journal of Applied Statistical Sciences, Vol. 21, No. 3, 239-259.

[16] Khan, H.M.R., Provost, S.B. and Singh, A. (2010), "Predictive inference from a two parameter Rayleigh life model given a doubly censored sample", Communications in Statistics - Theory and Methods, Vol. 39, 1237 - 1246.

[17] M. Shakil, M. Ahsanullah,, (2012). Review on Order Statistics and Record Values from $\mathrm{F}^{\alpha}$ Distributions. Pak.j.stat.oper.res. Vol. VIII No.1, 101-120.

[18] Merovci, F.,(2013). Transmuted Rayleigh distribution. Austrian Journal of Statistics, Vol. 42, No. 1, 21-31.

[19] Merovci, F., (2014). Transmuted generalized Rayleigh distribution, J. Stat. Appl. Pro. Vol. 3, No. 1, 9-20.

[20] Miklos Csorgo , Joseph L. Gastwirth, Ricardas Zitikis, (1998). Asymptotic confidence bands for the Lorenz and Bonferroni curves based on the empirical Lorenz curve; Journal of Statistical Planning and Inference. Vol. 74, 65-91.

[21] Rényi, Alfréd (1961). "On measures of information and entropy". Proceedings of the fourth Berkeley Symposium on Mathematics, Statistics and Probability, 1960. pp. 547-561.

[22] R Development Core Team, (2013). A Language and Environment for Statistical Computing, R Foundation for Statistical Computing. Vienna, Austria.

[23] Rayleigh, Lord. (1880). On the resultant of a large number of vibrations of the some pitch and of arbitrary phase. Philosophical Magazine and Journal of Science, 5-th Series, Vol. 10 (6), 73 - 78.

[24] Sanku Dey, Tanujit Dey and D. Kundu,. (2014). " Two-parameter Rayleigh distribution: Different methods of estimation " American Journal of Mathematical and Management Science, Vol. 33, No. 1, 55 - 74.

[25] Shaw. W. and Buckley. I. (2007). The alchemy of probability distributions: beyond Gram-Charlier expansions and a skewkurtotic-normal distribution from a rank transmutation map. Research report, King's College, London, U.K.

[26] Ullah, A. (1996). Entropy, divergence and distance measures with econometric applications. Journal of Statistical Planning and Inference. Vol. 49, 137-162.

[27] Yuzhu Tian, Maozai Tian \& Qianqian Zhu. (2014). Transmuted Linear Exponential Distribution: A New Generalization of the Linear Exponential Distribution, Communications in Statistics - Simulation and Computation, Vol. 43, No.10, 2661-2677.

[28] Yolanda M. Gómez, Heleno Bolfarine, Héctor W. Gómez. (2014). A New Extension of the Exponential Distribution, Revista Colombiana de Estadística, Vol. 37, No. 1, pp. $25-34$. 